EPJ Web of Conferences 41, 03023 (2013)

DOI: $10.1051 /$ epjconf/20134103023

(C) Owned by the authors, published by EDP Sciences, 2013

\title{
Charge density wave dynamics from ultrafast XUV ARPES
}

\author{
J.C. Petersen ${ }^{1,2}$, S. Kaiser ${ }^{2}$, N. Dean ${ }^{1}$, A. Simoncig ${ }^{2}$, H.Y. Liu ${ }^{2}$, A.L. Cavalieri ${ }^{2}$, C. Cacho ${ }^{3}$, I.C.E. \\ Turcu $^{3}$, E. Springate ${ }^{3}$, F. Frassetto ${ }^{4}$, L. Poletto ${ }^{4}$, S. S. Dhesi ${ }^{5}$, H. Berger ${ }^{6}$, and A. Cavalleri ${ }^{1,2}$ \\ ${ }^{1}$ Max Planck Research Dept. for Structural Dynamics, Centre for Free-Electron Laser Science, \\ University Of Hamburg, Hamburg, Germany \\ ${ }^{2}$ Clarendon Laboratory, Oxford University, Parks Road, Oxford, UK \\ ${ }^{3}$ Central Laser Facility, STFC Rutherford Appleton Laboratory, Harwell, UK \\ ${ }^{4}$ LUXOR, CNR-INFM, Padova, Italy \\ ${ }^{5}$ Diamond Light Source Ltd., Harwell, UK \\ ${ }^{6}$ Institute of Physics of Complex Matter, EPFL, Lausanne, Switzerland
}

\begin{abstract}
Ultrafast angle-resolved XUV photoemission reveals the time- and momentum-dependent electronic structure of 1T-TaS2, a hybrid Mott and chargedensity-wave insulator. Both electronic orderings melt well before the lattice responds, suggesting that electronic correlations play a role not just in the Mott localization but in the CDW ordering as well.
\end{abstract}

\section{Introduction}

Charge density waves (CDWs) underpin the electronic properties of many complex materials [1]. When electrons are uncorrelated, electron-phonon interactions drive CDW order through the Peierls mechanism [2]. This creates an insulating ground state in which both the charge density and the lattice structure are periodically modulated, opening a gap at the Fermi level. The low-energy excitations of the broken-symmetry state are collective modes in which the charge and lattice oscillate together. For some materials, physics beyond the Peierls model may be required to explain CDW formation, with strong electronic correlations playing a direct role [3]. One example is the layered dichalcogenide $1 T-\mathrm{TaS}_{2}$, in which a Mott gap opens as a commensurate CDW locks in to the lattice [4]. It is this Mott gap that yields an insulating ground state, while the CDW gap instead opens between occupied sub-bands at finite binding energy and momentum [5].

One way to query the nature of gaps in a complex insulator is to follow their evolution after photoexcitation [6]. Intense laser pulses destroy the ordered ground state and create a transient metallic phase, with the nature of the insulating phase reflected in the rate at which it melts. Mott gaps melt on the time scale of electronic hopping, whereas in one-dimensional Peierls CDW compounds, the gap at the Fermi level melts on the time scale of the breathing mode of the coupled charge and lattice [7]. In $1 T-\mathrm{TaS}_{2}$ the Mott gap at the Fermi level indeed melts within tens of femtoseconds [8], but dynamics of the CDW gap have not been measured because optical spectroscopy is not momentum-sensitive, and time-resolved ARPES experiments have used photon energies too low to see the momentum and binding energy in question. Here, we perform timeresolved ARPES with XUV pulses to observe the evolution of momentum-dependent electronic structure throughout the reconstructed Brillouin zone [9].

This is an Open Access article distributed under the terms of the Creative Commons Attribution License 2.0, which permits unrestricted use, distribution, and reproduction in any medium, provided the original work is properly cited. 


\section{XUV time-resolved ARPES}

In angle-resolved photoemission spectroscopy (ARPES), ultraviolet light is used to eject photoelectrons from a sample. In a two-dimensional material, the energy and exit angle of each photoelectron corresponds to the energy and momentum of the state from which it was removed. Analyzing the photoelectron distribution thus gives a map of occupied states in the material, revealing the momentum-dependent electronic band structure [10].

To study high-momentum electronic structure as it evolves on its natural timescale it is necessary to use ultrashort laser pulses with large photon energy. To create XUV pulses we focus a 30-fs amplified laser pulse into an Argon jet, generating high harmonics. We select the 13th harmonic $(20.4 \mathrm{eV})$ with a grating monochromator and focus it onto the sample to create photoelectrons. A train of 1.5-eV pulses from the same laser stimulates the sample. Varying the delay between excitation and photoemission pulses reveals the time evolution of electronic structure with a resolution of $24 \mathrm{fs}$ and $130 \mathrm{meV}$.

\section{Results}

Figure 1(a) shows the energy distribution of intensity at the centre and boundary of the reduced Brillouin zone, points known as $\Gamma$ and $\mathrm{M}$ respectively, as a function of time after the arrival of a 1.5$\mathrm{eV}$ photoexcitation pulse. Collapse of the Mott gap is confirmed by a rapid loss of intensity in the lower Hubbard band (LHB) immediately below the Fermi level $E_{f}$, accompanied by filling in of states at and above $E_{f}$. The original peaks then recover, followed by a rigid oscillation at the CDW amplitude mode frequency, agreeing with earlier results [3] for states near $E_{f}$ at $\Gamma$. Individual spectra (Fig. 1(b)) show that the CDW-derived splitting between occupied sub-bands is also lost, promptly within our time resolution of 24 fs. This effect is also visible in the series of snapshots of Fig. 1(c).

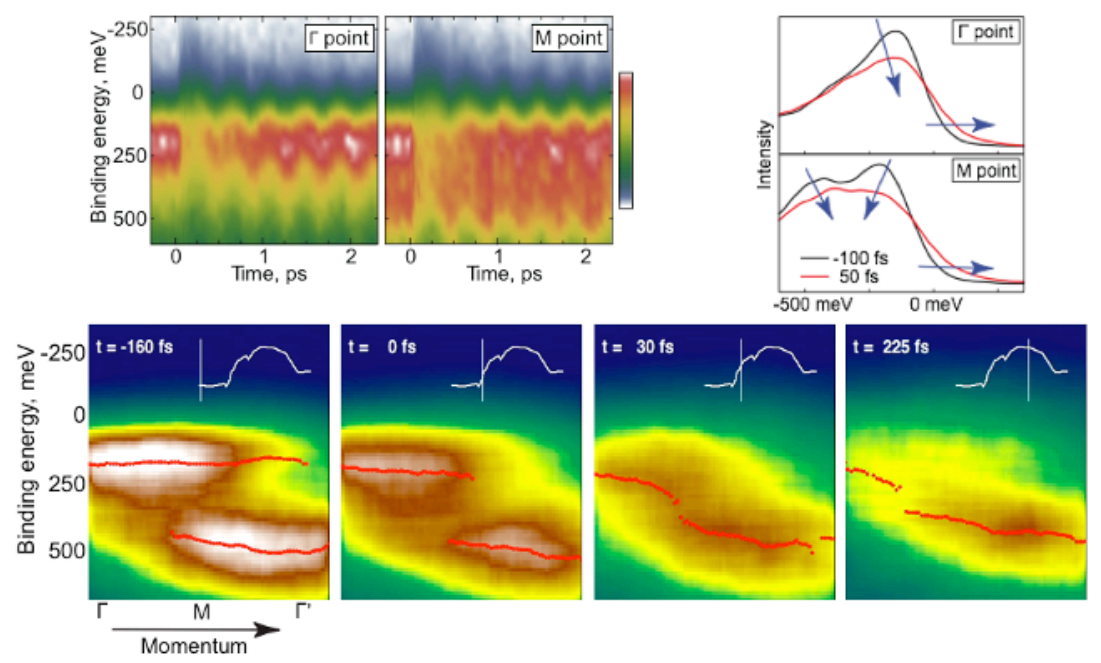

Fig. 1. (a) Energy distribution of photoemission intensity at the zone centre and boundary, as a function of time after photostimulation. (b) Photoinduced loss of the Mott gap (top) and CDW gap (bottom). (c) Snapshots of the momentum-dependent band structure.

At $\Gamma$, the lower-energy feature is an Umklapp or "shadow" band, consisting of higher-momentum states that were folded back into the first zone by the CDW transition. The intensity in this band responds more slowly, as illustrated in Fig. 2. This is consistent with a reduction in spectral weight brought about by incipient relaxation of the lattice distortion, on structural rather than electronic time scales [11]. 


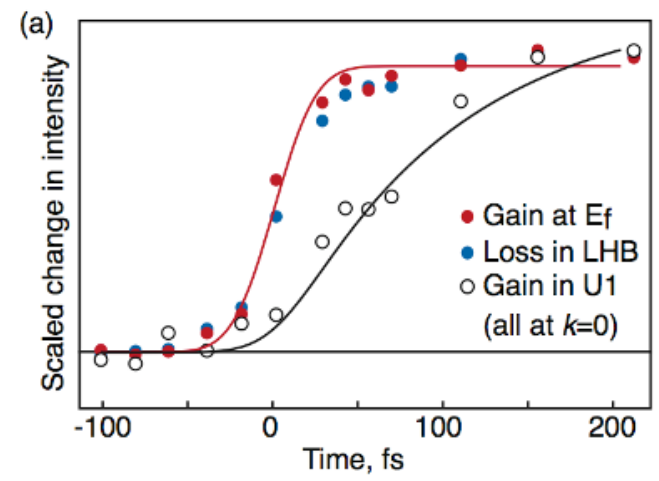

Fig. 2. Timescales for evolution of different spectral features.

\section{Discussion}

The fact that these different processes take place on distinct electronic and structural timescales gives new information about both the static band structure of 2D CDW materials, and the process by which they melt after ultrafast excitation. The prompt loss of the gap between sub-bands must be a response to the redistribution of charge, meaning that the broken symmetry of charge density in the CDW ground state is what originally caused it to open. By contrast, the shadow band intensity at the zone centre must reflect the periodic lattice distortion. At longer timescales, charge order and lattice distortions lock again, displaying the well-known modulations of spectral weight occurring at the frequency of the amplitude mode.

In summary, we have used time-resolved ARPES with femtosecond XUV pulses to study the relaxation of a charge density wave in photo-stimulated $1 T-\mathrm{TaS}_{2}$, measuring charge order at the edge of the Brillouin zone with sub-vibrational time resolution. CDW order, observed as a splitting between occupied sub-bands, melts well before the lattice responds, synchronously with Mott-gap collapse at $E_{f}$. The lattice then responds on its own time scale. These measurements highlight the importance of electron-electron correlations in localizing the charge on each site, challenging the view of the CDW as caused by Fermi surface nesting alone.

\section{References}

1. J. Wilson, F. DiSalvo, and S. Mahajan, Adv. Phys. 24, 117 (1975); P. Fazekas and E. Tosatti, Philos. Mag. B 39, 229 (1979); D. Jérome and H. J. Schulz, Adv. Phys. 31, 299 (1982); F. Clerc et al., Phys. Rev. B 74, 155114 (2006).

2. G. Grüner, Density Waves in Solids (Perseus, 2004).

3. T. Rohwer et al., Nature 471490 (2011); K. Rossnagel, J. Phys.: Cond.Matt. 23213001 (2011).

4. L. Perfetti et al., Phys. Rev. B 71153101 (2005).

5. K. Rossnagel and N. Smith, Phys. Rev. B 73073106 (2006)

6. A. Cavalleri et al., Phys. Rev. B 70161102 (2004); D. Polli et al., Nature Materials 61476 (2007); S. Wall et al., Nature Physics 7114 (2011); S. Wall et al., Phys. Rev. Lett. 103097402 (2009).

7. F. Schmitt et al., Science 3211649 (2008).

8. L. Perfetti et al., Phys. Rev. Lett. 97067402 (2006).

9. J. Petersen et al., Phys. Rev. Lett. 107177402 (2011).

10. A. Damascelli et al., Rev. Mod. Phys. 75473 (2003).

11. M. Eichberger et al., Nature 468799 (2010). 